\title{
The Burden of Chronic Kidney Disease on Developing Nations: A 21st Century Challenge in Global Health
}

\author{
Rachel A. Nugent $^{a}$ Sana F. Fathima ${ }^{c}$ Andrea B. Feigl ${ }^{c}$ Dorothy Chyung ${ }^{b}$ \\ ${ }^{a}$ Center for Global Development, Washington, D.C., and b Cornell Weill School of Medicine, New York, N.Y., USA; \\ 'University of Oxford, Oxford, UK
}

\section{Key Words}

Chronic diseases $\cdot$ Chronic kidney disease $\cdot$ Developing countries $\cdot$ Economic impact $\cdot$ Cost-effective intervention

\begin{abstract}
Chronic diseases present a significant challenge to 21st century global health policy. In developing nations, the growing prevalence of chronic diseases such as chronic kidney disease has severe implications on health and economic output. The rapid rise of common risk factors such as diabetes, hypertension, and obesity, especially among the poor, will result in even greater and more profound burdens that developing nations are not equipped to handle. Attention to chronic diseases, chronic kidney disease in particular, has been lacking, largely due to the global health community's focus on infectious diseases and lack of awareness. There is a critical need for funding in and to developing countries to implement more comprehensive, cost-effective, and preventative interventions against chronic diseases. This paper examines the epidemiology of chronic diseases, the growing prevalence of chronic kidney disease and its implications for global public health, and the associated health and economic burdens. Finally, a summary review of cost-effective interventions and funding needs is provided.
\end{abstract}

Copyright ๑ 2011 S. Karger AG, Basel

\section{Introduction to Chronic Diseases}

The pattern of disease burden in the 21st century has significantly shifted towards chronic diseases $(\mathrm{CDs})^{1}[1]$. Population aging and lifestyle-modifiable risk factors, accompanied by a decline in early-life infectious diseases, have resulted in the emergence of CDs as a major global health threat [2]. Both morbidity and mortality of CDs are rising, escalated by the increasing prevalence of pandemic health problems such as diabetes mellitus (DM) and cardiovascular disease (CVD). The expected increase in the burden of CDs is likely to have profound socioeconomic and public health consequences, especially in developing countries ${ }^{2}[3]$.

CDs are often considered to be a health problem endemic to the developed world, but the etiological link between infectious diseases and CDs and the global rise of $\mathrm{DM}, \mathrm{CVD}$, and nondiabetic chronic renal diseases have made CDs a primary health burden in developing countries [4]. Advances in medical innovation, focus on nutri-

\footnotetext{
In this context, CDs are defined as noncommunicable diseases that persist for long durations of time, such as CKD and CVD. The WHO describes CDs as type 2 diseases.

2 Developing countries, also referred to as low- and middle-income countries, are defined by criteria established by the World Bank.
}

\section{KARGER}

Fax +41613061234 E-Mail karger@karger.ch www.karger.com

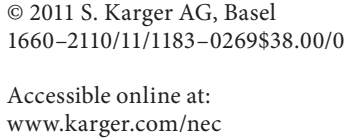

Rachel A. Nugent, PhD

Center for Global Development

1800 Massachusetts Ave NW, 3rd Floo

Washington, DC 20036 (USA)

Tel. +1 202416 4047, Fax +1 202416 0500, E-Mail rnugent@ cgdev.org 


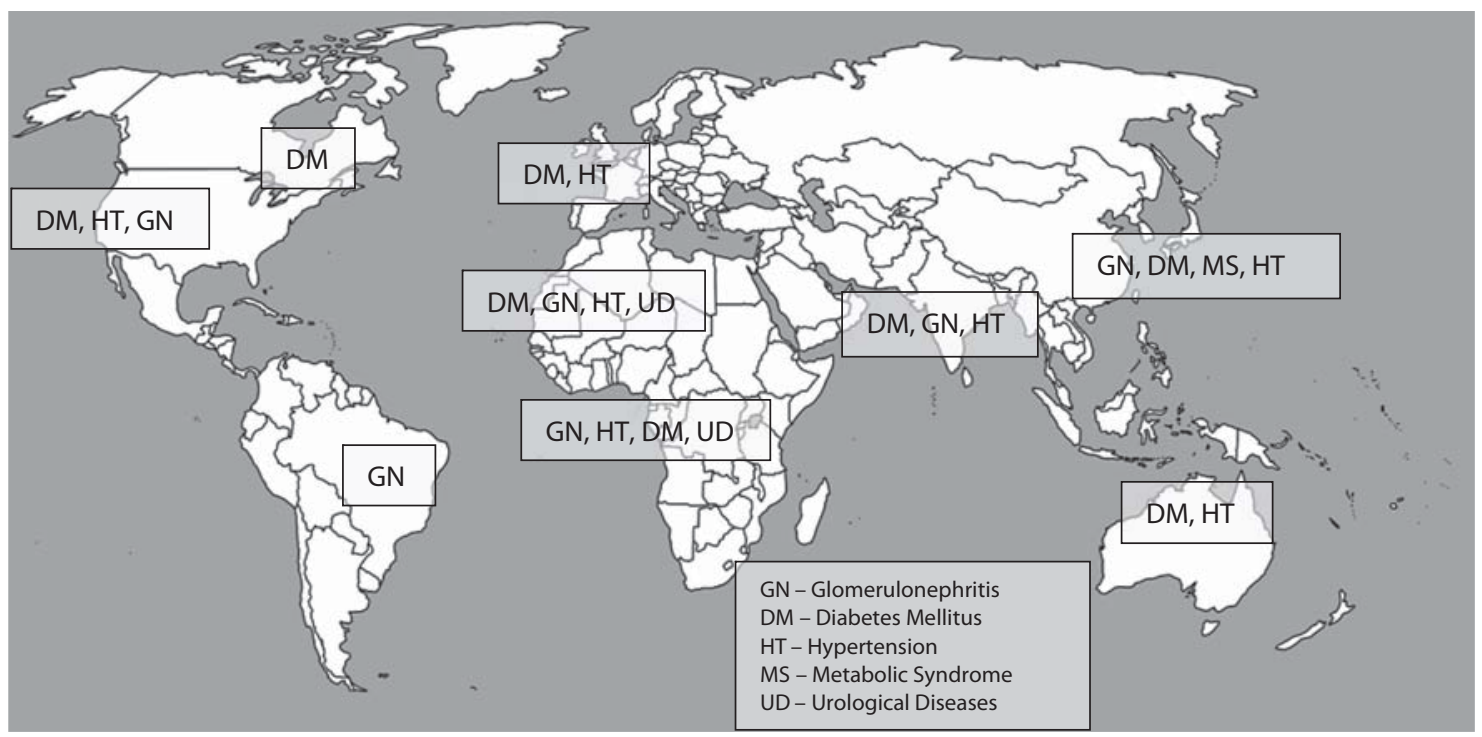

Fig. 1. Distribution of the main pathologies contributing to CKD across the world $[3,6,12,14,18,19,26,30,39$, $42,43,45,46,53,54]$.

tional health, economic improvement, and urbanization have resulted in a major surge in life expectancy and improvement in quality of life. These advances are countered by increased exposure to risk factors associated with CDs, such as unhealthy diets and lack of physical activity [5].

Among CDs, chronic kidney disease (CKD) is of particular significance and contributes heavily to the global CVD and end-stage renal disease (ESRD) [6, 7]. CKD ultimately progresses to ESRD, the rate of which is dependent on coexisting pathologies and risk factors [6]. Jungers et al. [8] found that the incidence of de novo CVD events in a non-ESRD cohort of CKD patients was $41 \%$ in men and $19 \%$ in women over a 10 -year span. As documented by Foley et al. [9], the prevalence of CVD in ESRD and end-stage CKD patients increases to $74 \%$. CKD is a burden not just for renal replacement therapy (RRT) demands but also for overall population health. Currently, CKD is the 12th highest cause of death and 17th highest cause of disability worldwide [6]. However, the rapid surge in diabetes and hypertension (HT), both of which are predicted to drive epidemics in CKD and CVD, will dramatically escalate this burden. CKD is expected to be a profound 21 st century medical challenge [10].

As the global health paradigm shifts towards CDs, the current development aid for health focus on infectious diseases [3] is no longer a sustainable approach in developing countries. The impact of CDs is overwhelming and there is a dire need for a greater alignment of funding with the burden of CDs. Coordinated and integrated action to target the growing prevalence of CDs will become essential in 21 st century global public health policy.

\section{Chronic Kidney Disease}

$\mathrm{CKD}^{3,4}$ is a complex and progressive condition [11] that arises from both noncommunicable diseases ${ }^{5}$ (fig. 1) and infectious diseases, such as malaria, schistosomiasis,

3 The National Kidney Foundation has established clinical criteria for CKD classification. According to its KDOQI guidelines, CKD is defined as (1) persistent glomerular filtration rate $<60 \mathrm{ml} / \mathrm{min} / 1.73 \mathrm{~m}^{2}$ for $\geq 3$ months with or without kidney damage or (2) kidney damage for $\geq 3$ months based on structural and functional anomalies of the kidney with or without decreased glomerular filtration rate, and manifested by either pathological abnormalities or abnormalities in the markers of kidney damage (i.e. composition of blood or urine or imaging tests).

4 KDOQI and KDIGO (Kidney Disease Improving Global Outcomes) guidelines recommend that estimates of glomerular filtration rate are the best overall evaluations of kidney function, though blood glucose tests, urine microscopy, and screening for hematuria, proteinuria, HT, and BMI are also incorporated in kidney disease diagnosis. Estimates of glomerular filtration rate values are estimated from prediction equations that incorporate serum creatinine levels and other variables including age, gender, race, and body size. Namely, the MDRD Study and Cockcroft-Gault equations are used for adults, and the Schwartz and Counahan-Barratt equations are used for children.

5 Noncommunicable diseases that contribute to CKD include glomerulonephritis, DM, HT, obstructive nephropathy, urological disease, and cystic kidney disease (fig. 1). 
Table 1. Population incidence of CKD stages 1-5 in select countries

\begin{tabular}{llllll}
\hline & Stage 1 & Stage 2 & Stage 3 & Stage 4 & Stage 5 \\
\hline Beijing study, China [14] & $2.9 \%$ & $2.2 \%$ & $1.3 \%$ & $0.05 \%$ & - \\
North Vietnam study, Vietnam [32] & - & - & & $3.6 \%$ & \\
\hline
\end{tabular}

HIV, and hepatitis B $[12,13]$. The use of herbal nephrotoxic medications, such as those containing aristolochic acid, also contributes to CKD (odds ratio $=2.19$ ) [14] Studies suggest that CKD may be a risk multiplier for infectious disease-associated mortality primarily in developing countries, which face comparatively higher infectious disease burdens [7]. However, infectious sources of CKD are still less common than chronic sources of CKD, especially in developing nations, where the many significant risk factors associated with kidney disease, such as HT, DM, dyslipidemia, and obesity, are becoming increasingly prevalent. It is characterized by 5 stages $^{6}$ of irreversible and impaired renal function, with the progression of renal function deterioration leading to ESRD, which is life-threatening without proper RRT intervention [15]. Stages 1 and 2 of CKD (covert CKD), as defined by the level of kidney function, are often asymptomatic and diagnosed by the presence of micro- and macroalbuminuria, which are markers for diffuse vascular damage and microinflammation associated with cardiokidney damage [13]. Covert CKD diagnosis is often a result of routine screening practices and generally targeted at high-risk patients [16].

Patients suffering from reduced kidney function, most often as a result of DM or HT, represent a global population that is highly vulnerable to CKD and ESRD and at great risk for many other CDs such as CVD $[6,17]$. The AusDiab study [18] showed that the prevalence of CKD markers in the Australian population was $12.1 \%$ - a result similar to that of the NHANES III study findings of $11 \%$ in the United States [10]. Studies in China suggest that between 12.1 [19] and 13\% [20] of the population have evidence of CKD and kidney damage markers. The profound impact of CKD on CDs and cardiovascular morbidity makes it especially important in the context of global disease, specifically in developing countries that have a mounting incidence of $\mathrm{CKD}$-associated risk factors $[21,22]$.

6 See Appendix: KDOQI stages of CKD.

\section{The Health Burden of CKD on Developing Countries}

Generally, the disease pattern shifts from infectious to chronic conditions as a country becomes more 'developed' [23]. However, in many of the poorest nations, the epidemiological shift to CDs as the major cause of morbidity and mortality is increasingly evident in spite of low overall indicators of development. Sixty percent of the 58 million deaths in 2005 were attributed to CDs, with 4 out of 5 deaths globally occurring in low- and middle-income countries (LMICs) [24]. By 2030, it is expected that 3 out of the 4 leading causes of death will be due to chronic conditions, indicating an alarming health burden [2]. Due to the escalating prevalence of CD risk factors, many developing countries now simultaneously bear the burden of both chronic and infectious diseases [25].

Currently, CVD and DM make up the largest contribution to the global CD burden. Developing countries such as India and China are major reservoirs for these CDs, with their incidence rising rapidly [26]. In China, between 1986 and 1999, age-specific death rates from circulatory diseases increased from 200 to $300 \%$ in those aged 35-44 years [27]. Ischemic heart disease and stroke mortality predictions between 1990 and 2020 include more than $100 \%$ increases in Latin America, the Middle East, and sub-Saharan Africa [28]. It is expected that the number of individuals with HT will increase from 972 million to 1.56 billion people by 2025 . In 2000 , the incidence of diabetes was estimated to be 171 million and WHO predictions expect this to increase to 366 million by 2030 [6]; 298 million of these individuals will be in developing countries [29].

Figure 2 offers a visual representation of the geographic burden of kidney disease in the world. Table 1 shows the population incidences of all stages of CKD in several developing countries. India, with the highest incidences of diabetes and HT in the world, is likely to face a catastrophic CKD/ESRD burden, with $25-40 \%$ of its population at risk [30]. Current estimates of CKD and ESRD in South Asia are approximately 800 and 100-200 per million people, respectively [26]. In China, the prevalence of CKD has been estimated to be $2-3 \%$ of the population or 10-15 million individuals [31]. 


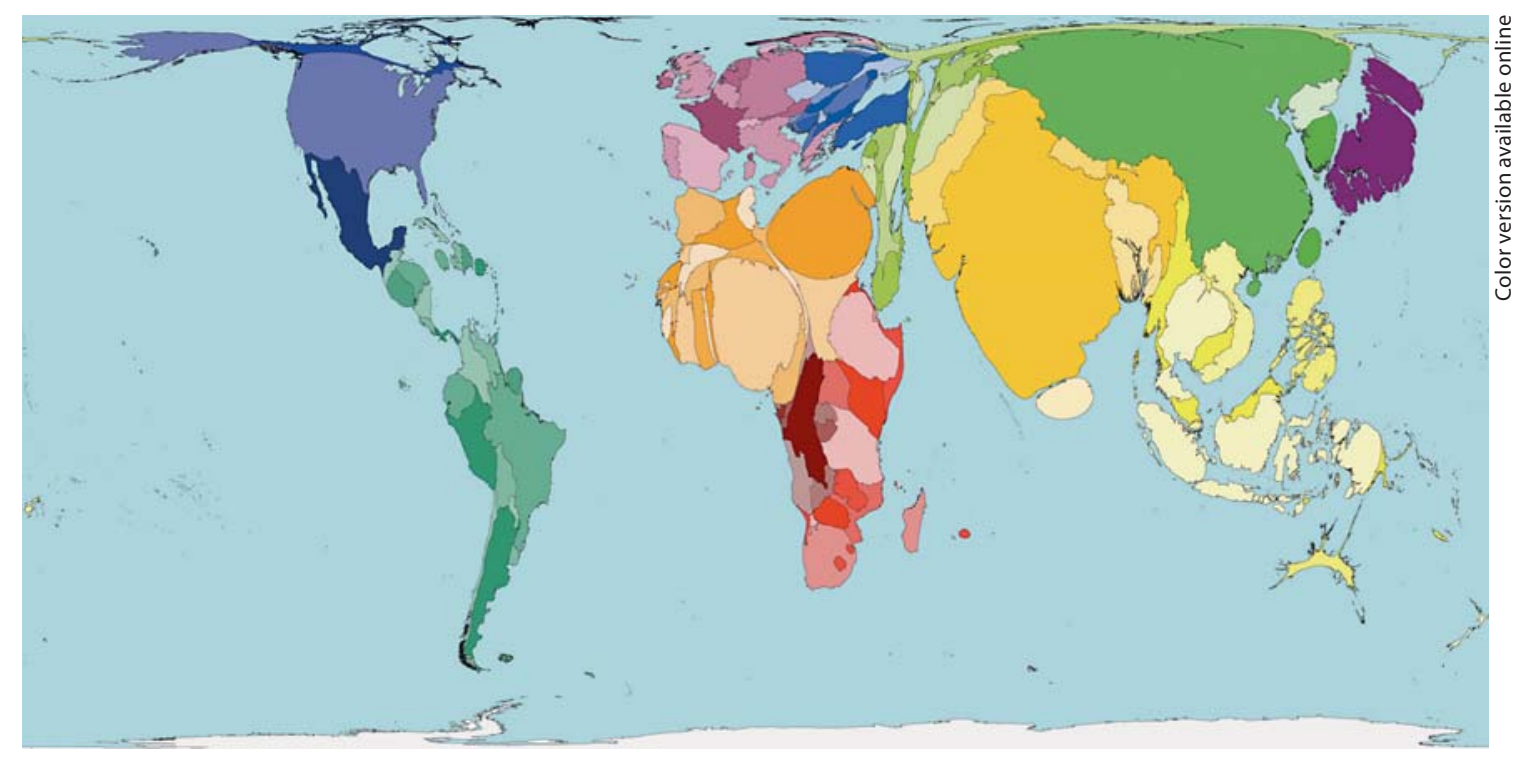

Fig. 2. Burden of mortality from kidney disease illustrated by relative country size (source: www.worldmapper. com). Territories are sized in proportion to the absolute number of people who died from kidney disease in 1 year. Copyright: SASI Group (University of Sheffield) and Mark Newman (University of Michigan).

The increase in CKD and its progression to end-stage renal failure worldwide are mainly a result of the rising global diabetes and HT pandemics $[21,22,32]$. The Demand Project, led by Parving et al. [33], tested 31,470 diabetics across 34 countries, and showed that $49 \%$ of the subjects had microalbuminuria, a key CKD/ESRD marker. The 2000 AusDiab study showed that $25.4 \%$ of Australian individuals with HT and diabetes had microalbuminuria [18]. A study in southern China showed CKD in $27.3 \%$ of patients with DM and HT, and 26.4\% of patients with metabolic syndrome or CVD [19]. A 2009 study conducted in the Congo showed that CKD was present in $44 \%$ of hypertensive patients and in $39 \%$ of diabetics [12]. The same study also showed a $16 \%$ CKD prevalence among obese patients and a $12 \%$ prevalence among HIV+ patients.

The progression of CKD to ESRD and its treatment depend heavily on the level of affluence of the country. Because of earlier stage mortality, LMICs have relatively low incidence rates of ESRD (150 per million people), as opposed to countries in North America and Europe [26]. Ninety percent of the 1.8 million people alive due to RRT reside in high-income countries [34]. Studies show that the incidence of ESRD has doubled in Europe and the USA over the past decade [19]. However, due to the rapid increase of several contributing risk factors, the number of ESRD patients in developing countries is expected to double to 2.1 million between 2001 and 2010 [35].

A survey across 10 Asian countries showed that the most common cause of ESRD in 9 out of 10 countries was diabetic nephropathy [13]. Diabetic nephropathy develops in 1 out of 3 diabetics worldwide, and is considered the leading cause of ESRD [13]. The remaining $66 \%$ of patients, mostly in developing countries, die from CVD prior to reaching ESRD, which contributes heavily to the burden of CVD ( $>30 \%$ of the global CD burden) [13]. However, estimated burdens of CKD in developing countries, most of which lack national renal disease registries, are often highly conservative representations of the overall national health burdens [36]. LMICs are not equipped to handle widespread screening for CKD, and surveillance studies are often limited to high-risk populations [16]. Furthermore, limited access to healthcare, lack of awareness, and limited capacity of health workers for CKD detection and prevention suggest that those in the lowest socioeconomic brackets are often unaware of any risk factors for CKD such as HT and DM. In Nepal, $47 \%$ of 1,243 subjects were newly diagnosed with HT [16]. In China, $75 \%$ of participants in a 2000 WHO study were newly diagnosed with diabetes [10]. In Pakistan, an estimated $70 \%$ of patients with high blood pressure and $50 \%$ of those with diabetes are unaware of their condition [37]. 
Table 2. Share of cause-specific deaths (age $<60$ years) by country income level [55]

\begin{tabular}{lllll}
\hline & $\begin{array}{l}\text { Low } \\
\text { income }\end{array}$ & $\begin{array}{l}\text { Lower- } \\
\text { middle } \\
\text { income }\end{array}$ & $\begin{array}{l}\text { Upper- } \\
\text { middle } \\
\text { income }\end{array}$ & $\begin{array}{l}\text { High } \\
\text { income }\end{array}$ \\
\hline (1) Communicable, maternal, perinatal and & & & & \\
$\quad$ nutritional conditions & $90 \%$ & $80 \%$ & $71 \%$ & $21 \%$ \\
(2) Chronic or noncommunicable conditions & $44 \%$ & $33 \%$ & $34 \%$ & $19 \%$ \\
(3) Injuries & $87 \%$ & $82 \%$ & $83 \%$ & $61 \%$ \\
\hline
\end{tabular}

\section{The Economic Burden of CKD on Developing Countries}

Economic growth studies suggest that the highest macroeconomic burden of CKD and other CDs falls on LMICs where high prevalence and the costs of treating disease create a greater proportional burden on the gross domestic product (GDP) (table 2). In 2005, CDs reduced GDP by an estimated $1 \%$ in Russia, and this number is expected to be over $5 \%$ by 2015 [2]. More recently, Abegunde and Stanciole [38] estimated the effects of 4 CDs on the economies of 23 high-burden developing countries using an economic growth model. These 23 countries account for approximately $80 \%$ of the total burden of CD mortality in developing countries. The study concluded that USD 84 billion of economic production will be lost due to heart disease, stroke, and diabetes in these countries between 2006 and 2015 [38].

Poverty and socioeconomic status are highly correlated with the common risk factors of CDs, including HT, DM, obesity, smoking, and dyslipidemia, making LMICs particularly vulnerable to the CD burden $[3,13]$. Prevalence of HT in Brazil is consistently higher (by 30-130\%) among the less educated, those in the lowest income bracket, and Afro-Brazilians [3]. Regions of low socioeconomic status are also less likely to have early detection and proper disease management [13]. In Jamaica, 59\% of people with CDs had illness-related financial difficulties, and a high proportion admitted to avoiding some medical treatment because of these financial barriers [39]. CD events can result in 'catastrophic' health costs, pushing people into poverty. For example, in China, out-of-pocket expenses for stroke management pushed $37 \%$ of patients and their families below the poverty line $-62 \%$ were without insurance [40].

Much of the economic burden of CDs can be attributed to direct medical expenditures associated with expensive and long-term treatment costs. In LMICs, public health care systems receive only $0.8-4 \%$ of the GDP, as opposed to $10-15 \%$ in developed countries [37]. Countries with high prevalence of DM are projected to spend up to $40 \%$ of their health budgets on diabetes and diabetes-associated management, while the average among developed nations ranges between 2.5 and $15 \%$ [10]. In 2007, the global estimate of health care expenditure for diabetes was between USD 232 and 421 billion, and was predicted to double by 2025 [10, 41]. However, only 10\% of these costs were borne by developing countries due to healthcare access, affordability, and treatment availability [6].

A similar trend can be seen with ESRD management, where developed countries dedicate more than $1 \%$ of the total health budget to the approximately $0.1 \%$ of the population that has ESRD [13]. However, ESRD management in LMICs is too expensive, and healthcare resources and budgets are unable to meet the burden of treatment. In Southeast Asia, RRT costs are more than 10 times the annual per capita income of approximately USD 400, and health insurance coverage is low or nonexistent for RRT/ CKD treatment $[32,37]$.

These high costs and lack of access to RRT are why less than $10 \%$ of patients in developing countries receive RRT [37]. The financial adjustments, reduced savings, decreased investment potential, and constrained educational attainment that families face due to the CD burden result in detrimental macroeconomic impacts. Overall, these effects are likely to translate into significantly decreased economic growth, and compounded over time would adversely affect disease management and control in already resource-poor settings [2].

\section{Cost-Effective Interventions}

In the developing world, CKD prevention programs are relatively nonexistent. Current interventions for CDs in LMICs have focused primarily on CVD, diabetes, chronic respiratory disorders, and cancer [42]. CKD, de- 
spite its numerous common modifiable risk factors and co-occurrence with many CDs, has not received the same kind of attention. This has been further exacerbated by lack of awareness, limited RRT availability, late referral of patients, and decreased capacity for CKD detection and prevention among healthcare practitioners [12].

Studies from developed countries show that mortality risk for CDs can be reduced by $75 \%$ through health interventions and the incidence can be reduced by up to $58 \%$ through lifestyle interventions [43]. Cost-effective population-based health intervention is based mainly on three major risk factors: poor diet, lack of physical activity, and tobacco use. This includes tobacco restrictions, salt reduction, elimination of caloric sweeteners, and fat substitution. For example, an increase in the tobacco price of 33\% would cost USD 2-85 per Disability Adjusted Life Year $^{7}$ Added $^{8}$ (DALYA) [44]. Similarly, replacing trans fats with polyunsaturated fats would cost USD 25-75 per DALYA, and salt reduction can be achieved for less than USD 200 per DALYA. These interventions could extend to caloric sweeteners such as high-fructose corn syrup, which contribute to the obesity and metabolic syndrome epidemics in LMICs [45]. Cost-effective lifestyle and behavioral changes can also be achieved on a population basis through legislation, government influence, manufacturing changes, mass education campaigns and advertising bans $[16,44]$.

Arguably the most cost-effective solution for CKD and ESRD treatment in LMICs is renal transplantation, but this is heavily limited by cadaver availability and resources [26]. Thus, an emphasis on prevention planning and early detection of CKD on a long-term basis is the only practical and cost-effective solution for developing countries that cannot afford the burden of expensive treatment associated with CDs. Individual level interventions can be done through the screening of (high-risk) people and providing multidrug regimens of aspirin, ACE inhibitors, statin, calcium channel or $\beta$-blockers for early treatment [44]. Primary and secondary prevention using these medicines is projected to cost USD 350-825 per DALYA in LMICs. Potentially, a combination of these drugs with a diuretic and folic acid in the form of a single tablet, a 'polypill', could be used - this approach is still being tested in clinical trials [44]. Several countries have adopted prevention planning in their public health initia-

\footnotetext{
A disability-adjusted life year (DALY) is a year of 'healthy' life that is lost to either death or disability.

8 A DALYA is a year of 'healthy life' that is added and that would otherwise be lost to either death or disability.
}

tives. One such example can be found in Uruguay, which established a program of preventive education for clinicians and patients to reduce the incidence and decrease health expenditures for kidney failure [25].

Currently, screening for CKD is accepted practice only in patients with HT or diabetes [46, 47]. A survey conducted on 65,604 people in Norway indicated that a highrisk screening model targeted at this population would identify less than half of those with CKD [48]. This suggests that screening of the general population should be proposed and implemented. Furthermore, early detection of CDs such as CKD, which are relatively asymptomatic, has a reasonable potential to have a positive impact on the outcome of the disease [16]. Studies in developed countries on the cost-effectiveness of tertiary prevention of CKD by treatment of HT, albuminuria, and use of renin-angiotensin system inhibitors have shown that early intervention appears to be more cost-effective than late intervention [49, 50]. Preventive education and early screening and identification would alleviate the economic burden of more advanced CKD and RRT treatments [16]. These alternatives and preemptive actions would also serve as low-cost solutions in managing the global trends of CKD and ESRD prevalence.

\section{Funding Needs}

International donors are an important source of health care financing in LMICs. However, donors have almost completely neglected CDs, especially CKD. Of USD 21.7 billion in donor assistance for heath in 2007, CDs received less than 3\% [56]. This is consistent with several earlier analyses showing that donor assistance for health is heavily skewed towards infectious diseases, especially HIV/AIDS [51]. However, recent trends are progressing in the right direction. Global donor funding for CVD and other CDs increased from USD 236 million in 2004 to over USD 750 million in 2008 in real terms [56].

The largest proportional increase in donor funding comes from the private sector (mainly nonprofit) which collectively contributed over USD 267 million in 2008, constituting an almost 2,000\% increase in private sector funding since 2004 [56]. CD funding from multilateral organizations increased from USD 175 million to USD 305 million, almost doubling between 2004 and 2008 . Funding contributions from research institutions, public health organizations, and disease membership associations made up less than 3\% of CD funding in all years between 2004 and 2008 [Nugent and Feigl, unpubl. data]. 
This diversification of funding is a positive trend, particularly because of the expertise and in-kind resources that private funders can bring; however, international and national governmental organizations should be encouraged to become more active in order to ensure accountability in global health equity. Despite the inadequate amount of funds, improvements can be made in the management and allocation of current appropriations. This limited and disproportionate funding focus on high-burden CDs is no longer a sustainable option, and funding must not only be increased but also designed to inclusively target the lowest socioeconomic regions in the world.

\section{Time for Action}

A comprehensive, integrated approach to managing the CD burden, with effective intervention tailored specifically to LMICs is essential to 21st century global health policy. There is a dire need for a greater alignment of global disease funding with CD burden, transparency of projects, data availability, and better sharing of knowledge for more targeted, effective, and coordinated activities to combat the global problem of CDs. Health interventions and international efforts must take the emerging burden of CKD among other CDs into account, especially as the overall population health burden due to CKD and its co-occurring pathologies are increasingly too great for many countries.

Aside from the specific cost-effective strategies to decrease the $\mathrm{CD}$ burden already mentioned, there are many more general strategies that can be implemented. In addressing the issues around common risk factors of CDs such as $\mathrm{CKD}, \mathrm{DM}$, and CVD, the preventive and primary health care systems of developing countries must be strengthened and unified. There is also a desperate need for public awareness and advocacy towards lifestyle changes and disease management, increased training and availability of medical practitioners, and for expanded surveillance and screening systems for CDs. The Disease Control Priorities Project offers such a recommendation, supporting the establishment of international centers of excellence for CKD that can work in tandem with centers of CVD and diabetes [52]. The International Society of Nephrology and Kidney Disease Improving Global Outcomes has responded to the challenging CKD burden by forming collaborations with societies such as the International Diabetes Federation (IDF), WHO, and the World Bank to influence global and national health decision-making as regards the growing burden of CKD and $\mathrm{CD}$. Other similar initiatives must now be brought forth and implemented $[10,45]$. The time to act is now.

Appendix. KDOQI stages of CKD

\begin{tabular}{lll}
\hline Stage & Description & $\begin{array}{l}\mathrm{GFR} \\
\mathrm{ml} / \mathrm{min} / 1.73 \mathrm{~m}^{2}\end{array}$ \\
\hline 1 & Kidney damage with normal or $\uparrow \mathrm{GFR}$ & $\geq 90$ \\
2 & Kidney damage with mild $\downarrow$ GFR & $60-89$ \\
3 & Moderate $\downarrow$ GFR & $30-59$ \\
4 & Severe $\downarrow$ GFR & $15-29$ \\
5 & Kidney failure, ESRD & $<15$ \\
& & (or dialysis) \\
\hline
\end{tabular}

CKD is defined as (1) persistent GFR $<60 \mathrm{ml} / \mathrm{min} / 1.73 \mathrm{~m}^{2}$ for $\geq 3$ months with or without kidney damage or (2) kidney damage for $\geq 3$ months based on structural and functional anomalies of the kidney with or without decreased GFR [11]. GFR = Glomerular filtration rate.

\section{References}

1 World Health Organization: The Global Burden of Disease: 2004 Update. Geneva, WHO, 2008.

2 World Health Organization: Preventing Chronic Diseases: A Vital Investment. Geneva, WHO, 2005.

3 World Bank: Addressing the Challenge of Non-Communicable Diseases in Brazil, 2005.
4 Murray C, Lopez A: The Global Burden of Disease: A Comprehensive Assessment of Mortality and Disability from Diseases, Injuries, and Risk Factors in 1990 and Projected to 2020. Cambridge, Harvard University Press, 1996

5 World Health Organization: The World Health Report 2002 - Reducing Risks, Promoting Healthy Life. Geneva, WHO, 2002.

-6 Codreanu I, et al: Prevention programmes of progressive renal disease in developing nations. Nephrology (Carlton) 2006;11:321328.
7 Levey AS, et al: Chronic kidney disease as a global public health problem: approaches and initiatives - a position statement from Kidney Disease Improving Global Outcomes. Kidney Int 2007;72:247-259.

8 Jungers $\mathrm{P}$, et al: Incidence and risk factors of atherosclerotic cardiovascular accidents in predialysis chronic renal failure patients: a prospective study. Nephrol Dial Transplant 1997;12:2597-2602. 
$\checkmark 9$ Foley RN, et al: Clinical and echocardiographic disease in patients starting endstage renal disease therapy. Kidney Int 1995; 47:186-192.

10 Atkins RC: The epidemiology of chronic kidney disease. Kidney Int Suppl 2005;94:S14S18.

11 National Kidney Foundation: K/DOQI clinical practice guidelines for chronic kidney disease: evaluation, classification, and stratification. Am J Kidney Dis 2002;39(suppl 1):S1-S266

$\checkmark 12$ Sumaili EK, et al: High prevalence of undiagnosed chronic kidney disease among atrisk population in Kinshasa, the Democratic Republic of Congo. BMC Nephrol 2009;10: 18.

13 Hossain MP, et al: CKD and poverty: a growing global challenge. Am J Kidney Dis 2009; 53:166-174.

14 Zhang L, et al: Prevalence and factors associated with CKD: a population study from Beijing. Am J Kidney Dis 2008;51:373-384.

15 Vachvanichsanong P, Dissaneewate P, McNeil E: Childhood chronic kidney disease in a developing country. Pediatr Nephrol 2008; 23:1143-1147.

-16 Perico N, et al: Screening for chronic kidney disease in emerging countries: feasibility and hurdles. Nephrol Dial Transplant 2009; 24:1355-1358.

17 Levey AS, et al: Definition and classification of chronic kidney disease: a position statement from Kidney Disease: Improving Global Outcomes (KDIGO). Kidney Int 2005;67: 2089-2100.

18 Chadban SJ, et al: Prevalence of kidney damage in Australian adults: The AusDiab kidney study. J Am Soc Nephrol 2003;14(suppl 2):S131-S138

$\checkmark 19$ Chen W, et al: Prevalence and risk factors associated with chronic kidney disease in an adult population from southern China. Nephrol Dial Transplant 2009;24:12051212 .

20 Zhang L, et al: Community-based screening for chronic kidney disease among populations older than 40 years in Beijing. Nephrol Dial Transplant 2007;22:1093-1099.

21 Yach D, et al: The global burden of chronic diseases: overcoming impediments to prevention and control. JAMA 2004;291:26162622 .

-22 Beaglehole R, Yach D: Globalisation and the prevention and control of non-communicable disease: the neglected chronic diseases of adults. Lancet 2003;362:903-908.

23 Ezzati M, et al: Rethinking the 'diseases of affluence' paradigm: global patterns of nutritional risks in relation to economic development. PLoS Med 2005;2:e133.
24 Monteiro CA, et al: Obesity and inequities in health in the developing world. Int J Obes Relat Metab Disord 2004;28:1181-1186.

25 World Bank: Public Policy and the Challenge of Chronic Non-Communicable Disease. Washington, 2007.

26 Agarwal SK, Srivastava RK: Chronic kidney disease in India: challenges and solutions. Nephron Clin Pract 2009;111:c197-c203, discussion c203.

27 Bumgarner R: Non-communicable disease issues and options revisited. Soc Prev Med 2004;38:202-210.

28 Leeder S, Raymond S, Greenberg H: A Race against Time: The Challenge of Cardiovascular Disease in Developing Economies. New York, Columbia University, 2004.

29 International Diabetes Federation: Diabetes Atlas, ed 2. Brussels, International Diabetes Federation, 2003.

30 Srinath Reddy K, et al: Responding to the threat of chronic diseases in India. Lancet 2005;366:1744-1749.

31 World Bank: PovertyNet, Washington, 2010

32 Ito J, et al: Impact and perspective on chronic kidney disease in an Asian developing country: a large-scale survey in North Vietnam. Nephron Clin Pract 2008;109:c25c32.

33 Parving HH, Lewis JB, Ravid M: Prevalence and Risk Factors for Microalbuminuria in Type 2 Diabetic Patients: A Global Perspective. Copenhagen, Steno Diabetes Center, 2004.

34 Remuzzi G, Weening J: Albuminuria as early test for vascular disease. Lancet 2005;365 556-557.

35 Lysaght MJ: Maintenance dialysis population dynamics: current trends and long-term implications. J Am Soc Nephrol 2002; 13(suppl 1):S37-S40.

36 Arogundade FA, Barsoum RS: CKD prevention in Sub-Saharan Africa: a call for governmental, nongovernmental, and community support. Am J Kidney Dis 2008;51:515-523.

37 Jafar TH: The growing burden of chronic kidney disease in Pakistan. N Engl J Med 2006;354:995-997.

38 Abegunde D, Stanciole A: An Estimation of the Economic Impact of Chronic Noncommunicable Diseases in Selected Countries. Geneva, WHO, 2006

39 Henry-Lee A, Yearwood A: Protecting the Poor and the Medically Indigent under Health Insurance: A Case Study of Jamaica. Bethesda, Partnerships for Health Reform Project, 1999.
40 Heeley E, et al: Role of health insurance in averting economic hardship in families after acute stroke in China. Stroke 2009;40:21492156.

41 International Diabetes Federation: Diabetes Atlas, ed 3. Brussels, International Diabetes Center, 2007.

42 Barsoum RS: Chronic kidney disease in the developing world. N Engl J Med 2006;354: 997-999.

43 Puska P: Successful prevention of non-communicable diseases: 25 year experiences with North Karelia Project in Finland. Public Health Med 2002;4:5-7.

44 Gaziano T, Galea G, Reddy K: Scaling up interventions for chronic disease prevention: the evidence. Lancet 2007;370:1939-1946.

45 Iseki K: Metabolic syndrome and chronic kidney disease: a Japanese perspective on a worldwide problem. J Nephrol 2008;21:305312.

46 Williams B, et al: British Hypertension Society guidelines for hypertension management 2004 (BHS-IV): summary. BMJ 2004;328: 634-640.

47 Tseng K: Standards of medical care in diabetes - 2006. Diabetes Care 2006;29(suppl 1):S4-S42.

48 Hallan S, et al: Screening strategies for chronic kidney disease in the general population: follow-up of cross sectional health survey. BMJ 2006;333:1047.

49 Ruggenenti P, et al: Ramipril prolongs life and is cost effective in chronic proteinuric nephropathies. Kidney Int 2001;59:286-294.

50 Palmer AJ, et al: An economic evaluation of the Irbesartan in Diabetic Nephropathy Trial (IDNT) in a UK setting. J Hum Hypertens 2004;18:733-738

51 Yach DY, Hawkes C: Towards a WHO LongTerm Strategy for Prevention and Control of Leading Non-Communicable Diseases. Geneva, WHO, 2004

52 Jamison D, Mosley W, Measham A: Disease Control Priorities in Developing Countries. Washington, World Bank, 2006.

53 Coresh J, et al: Prevalence of chronic kidney disease and decreased kidney function in the adult US population: Third National Health and Nutrition Examination Survey. Am J Kidney Dis 2003;41:1-12.

54 Kher V: End-stage renal disease in developing countries. Kidney Int 2002;62:350-362.

55 Mathers CD: Towards valid and comparable measurement of population health. Bull World Health Organ 2003;81:787-788.

56 Nugent R, Feigl A: Where Have All the Donors Gone? Scarce Donor Funding for NonCommunicable Diseases. CGD Working Paper 228. Washington, Center for Global Development, 2010. 


\section{Editorial Comment}

Meguid El Nahas, Sheffield

The review by Nugent from the Center for Global Development and her colleagues highlights issues related to the impact of chronic kidney disease (CKD) on health in emerging economies. Such countries are the subject of a triple hit that leads to the development of CKD and impacts on population health and survival, poverty, infectious (communicable) disease as well as westernization with the sharp rise in noncommunicable chronic disease. The latter, as highlighted in the review, includes obesity with its impact on the development of diabetes, hypertension and cardiovascular disease (CVD). People in developing countries also live longer and this is associated with increased life course exposure to risk factors that lead to CKD and CVD. Cost-effective detection and management approaches are also advocated. They have to be adjusted to meet the local needs and means. They also have to take into consideration access to care and related cost. The 21st century with advances in telecommunication may open new and exciting opportunities to access healthcare in emerging countries. After all, before too long most people in those countries will have a mobile phone, but few will have access to a doctor! Perhaps, Mobile Health can be one way forward... 